$$
\mathrm{d} s=0
$$

as it should be for this formal change.

Equation (1) would lead to the obviously wrong result $\mathrm{d} s \neq 0$, whereas equation (2), which is entirely unaffected by the change of the gauge of $\varphi$, again yields the correct result, equation (4).

$$
\begin{aligned}
& \text { S. R. DE GRoOT } \\
& \text { P. MAZUR } \\
& \text { H. A. TOLHOEK }
\end{aligned}
$$

Institute for Theoretical Physics, University, Utrecht.

${ }^{1}$ Kemp, P. H., Nature, 170, 1028 (1952).

Groot, S. R. de, and Tolhoek, H. A., Proc. Roy. Netherlands Acad. B, 54, 42 (1951).

${ }^{3}$ Mazur, P., and Prigogine, T., Mém. Acad. Roy. Blg., Cl. Sci. (in the press).

${ }^{1}$ Prigogine, I., J. Chim. Phys., 43, C 80 (1952).

s Prigogine, I., Mazur, P., and Defay, R., J. Chim. Phys. (in the press).

- Guggenheim, E. A., J. Phys. Chem., 33, 842 (1929); "Thermodynamics" (especially chapter 10, N̉orth Holland Publishing Co., Amsterdam, 1949).

'Tolhoek, H. A., and Groot, S. R. de, Physica, 18, 780 (1952).

${ }^{8}$ Prigogine, I., Physica, 15, 272 (1949).

The above letter by Prof. S. R. de Groot and his co-workers has directed my attention to an error in my earlier communication ${ }^{1}$, but has also made more obvious the fundamental error in their own paper ${ }^{2}$.

In that paper they considered a medium showing a bulk motion and in which chemical reactions and flows of energy, matter and electricity were occurring. Their treatment was in effect a detailed consideration of the thermodynamics of a system consisting of an elementary volume of that medium. In virtue of the flows of matter, this system is an open one, and the work which it can perform is thus of four kinds : volume work, osmotic work, electrical work arising from changes in the concentrations of the charged components, and electrical work arising from changes in the electrical potential of the system. Using Gibbs's equation, we thus have, expressing the extensive properties in terms of unit mass :

$$
T \mathrm{~d} s=\mathrm{d} u+p \mathrm{~d} v-\sum_{k} \mu k \mathrm{~d} c_{k}-\sum_{k} e_{k} \varphi \mathrm{d} c_{k}-e \mathrm{~d} \varphi,(1)
$$

or, introducing the electrochemical potentials :

$$
T \mathrm{~d} s=\mathrm{d} u+p \mathrm{~d} v-\sum_{k} \tilde{\mu}_{k} \mathrm{~d} c k-e \mathrm{~d} \varphi .
$$

Equation (2) differs from that of my earlier communication, in which the last term on the right was erroneously omitted, and now leads to profoundly different conclusions.

The question of the validity of Gibbs's equation in relation to the present problem is of extreme importance, but is not the point now at issue. It may be noted, however, that de Groot and Tolhoek in their original paper ${ }^{2}$ made use of Gibbs's equation without any hesitation.

If the formal change d $\varphi$ in the gauge of $\varphi$ is now made at constant volume and composition, the specific internal energy $u$ changes by an amount $e d \varphi$, corresponding to the formal change in the potential energy which has now occurred; all other terms on the right of equation (1) or of equation (2) are zero except the last, $-e \mathrm{~d} \varphi$. Hence we have the necessary result $\mathrm{d} s=0$.

Instead of equations (1) or (2), de Croot and his co-workers use for Gibbs's equation :

$$
T \mathrm{~d} s=\mathrm{d} u+p \mathrm{~d} v-{ }_{k} \mu_{k} \mathrm{~d} c
$$

and the same argument shows that, contrary to their claim, the formal change in the gauge of $\varphi$ now in- correctly yields $\mathrm{d} s \neq 0$. Thus it is equation (3) and its various other forms (all identical in physical content) which are at fault, not equation (1). In fact, equation (3), which contains no electrical terms whatever, cannot possibly apply to the system under consideration.

When equation (1) is employed in place of equation (3) in the study of the system under consideration, it is found that the entropy production can be written as the sum of a product of thermodynamic forces and fluxes. Furthermore, with a suitable choice of forces and fluxes, the quantities $\mu_{k}$ and $e_{k}$ appear in the expression for the entropy production only in the combinations $\tilde{\mu}^{k}$, provided that the condition $e=0$ applies. This result implies that $\varphi$ can be measured separately from the $\mu_{k}$ only if $e \neq 0$, that is, if a space charge is present in the system.

These conclusions are now identical with those of de Groot and Tolhoek, although these authors employed an incorrect form of Gibbs's equation. In arriving at these conclusions, however, they employed only the force equation (equation of motion for the kth component), which is not affected by any errors made in Gibbs's equation. The correct form, equation (1), of the latter now allows the same conclusions to be obtained directly from the entropy balance equation.

37 Friern Court,

Friern Barnet Lane,

Peter H. Kemp

London, N.20.

${ }^{1}$ Kemp, P. H., Nature, 170, 1028 (1952). Groot, S. R. de, and Tolhoek, H. A., Proc. Roy. Netherlands Acad.,
B, 54, 42 (1951).

\section{Structure of Meta-aminophenol}

A SINGLE crystal of meta-aminophenol was previously studied ${ }^{1}$ by means of rotation photographs only, whereby the identification of spots remains doubtful. We used the Weissenberg method and identified the spots by the method of Schneider ${ }^{2}$. We also studied the optical properties of this crystal.

The crystals were obtained by slow evaporation of alcohol solution. The crystal is orthorhombic with the following axial data : $a=6 \cdot 31 \mathrm{~A} ., b=11 \cdot 12 \mathrm{~A}$., $c=8.60 \mathrm{~A}$.; with density (observed) $1 \cdot 195$, $\eta$ the number of molecules per unit cell is 4. With these data, density was calculated to be $\mathbf{1} 206$.

The crystal was found to be optically negative with the values of refractive indices $\gamma, \beta$ and $\alpha$ along $a$-, $b$ - and $c$-axes, respectively: $\gamma=1 \cdot 638, \beta=1 \cdot 625$ and $\alpha=1.505$, and $v_{a}=15^{\circ} 30^{\prime}$, where $v_{a}$ is half the optic-axial angle.

If the molecules form a parallelism among themselves then the plane of the benzene ring will have a least value of refractive index perpendicular to the plane of the ring.

This result shows that the plane of the benzene ring is closer to the $\alpha$-and $\beta$-directions than to the $\gamma$-direction.

Over-exposed oscillation photographs about the three crystallographic axes were taken in a Weissenberg goniometer using Ni-radiation. The spots were identified by drawing the requisite Weissenberg charts by the method of Schneider ${ }^{2}$. There are no absent spectra of $(h k i)$. When $l$ is odd $(h 0 l)$ is absent, but not $(0 K l)$. The probable space group is $P_{m a}\left(C_{2 v}^{4}\right)$.

Physies Department,

University of Calcutta. July 9.

M. N. Dutr

${ }^{1}$ Caspari, W. A., Phil. Mag., (7), 4, 1276 (1928).

'Schneider, W., Z. Krystallog., 69, 41 (1928). 